Journal for ImmunoTherapy of Cancer

\title{
Immune checkpoint inhibitors in patients with pre-existing psoriasis: safety and efficacy
}

\author{
Briana Rose Halle (D) , ${ }^{1}$ Allison Betof Warner (D) ,2 Farzana Y Zaman, ${ }^{3}$ \\ Andrew Haydon, ${ }^{4}$ Prachi Bhave, ${ }^{5}$ Anna K Dewan, ${ }^{6}$ Fei Ye, ${ }^{7}$ Rebecca Irlmeier, ${ }^{7}$ \\ Paras Mehta, ${ }^{2}$ Nicholas R Kurtansky (D) , ${ }^{2}$ Mario E Lacouture, ${ }^{2}$ Jessica C Hassel, ${ }^{8}$ \\ Jacob S Choi, ${ }^{9}$ Jeffrey A Sosman, ${ }^{9}$ Sunandana Chandra, ${ }^{9}$ Tracey S Otto, ${ }^{10,11}$ \\ Ryan Sullivan (D) , ${ }^{11}$ Meghan J Mooradian (D) , ${ }^{11}$ Steven T Chen, ${ }^{12}$ \\ Florentia Dimitriou, ${ }^{13,14}$ Georgina Long, ${ }^{13,15}$ Matteo Carlino, ${ }^{13,16}$ \\ Alexander Menzies, ${ }^{13,15}$ Douglas B Johnson, ${ }^{17}$ Veronica M Rotemberg ${ }^{2}$
}

To cite: Halle BR, Betof Warner A, Zaman FY, et al. Immune checkpoint inhibitors in patients with pre-existing psoriasis: safety and efficacy. Journal for ImmunoTherapy of Cancer 2021;9:e003066. doi:10.1136/jitc-2021-003066

- Additional supplemental material is published online only. To view, please visit the journal online (http://dx.doi.org/10. 1136/jitc-2021-003066).

$\mathrm{BRH}$ and $\mathrm{ABW}$ contributed equally.

Accepted 31 August 2021
Check for updates

(C) Author(s) (or their employer(s)) 2021. Re-use permitted under CC BY-NC. No commercial re-use. See rights and permissions. Published by BMJ.

For numbered affiliations see end of article.

Correspondence to

Briana Rose Halle;

briana.r.halle@vanderbilt.edu

\section{ABSTRACT}

Background Immune checkpoint inhibitors (ICls) are approved to treat multiple cancers. Retrospective analyses demonstrate acceptable safety of ICls in most patients with autoimmune disease, although disease exacerbation may occur. Psoriasis vulgaris is a common, immunemediated disease, and outcomes of ICI treatment in patients with psoriasis are not well described. Thus we sought to define the safety profile and effectiveness of ICls in patients with pre-existing psoriasis.

Methods In this retrospective cohort study, patients from eight academic centers with pre-existing psoriasis who received ICI treatment for cancer were evaluated. Main safety outcomes were psoriasis exacerbation and immune-related adverse events (irAEs). We also assessed progression-free survival (PFS) and overall survival. Results 0 f 76 patients studied (50 $(66 \%)$ male; median age 67 years; 62 (82\%) with melanoma, $5(7 \%)$ with lung cancer, $2(3 \%)$ with head and neck cancer, and 7 (9\%) with other cancers; median follow-up 25.1 months (range $=0.2-99$ months)), 51 (67\%) received antiPD-1 antibodies, 8 (11\%) anti-CTLA-4, and 17 (22\%) combination of anti-PD-1/CTLA-4. All patients had preexisting psoriasis, most frequently plaque psoriasis (46 patients (61\%)) and $15(20 \%)$ with psoriatic arthritis. Forty-one patients $(54 \%)$ had received any prior therapy for psoriasis although only two (3\%) were on systemic immunosuppression at ICI initiation. With ICI treatment, 43 patients (57\%) experienced a psoriasis flare of cutaneous and/or extracutaneous disease after a median of 44 days of receiving $\mathrm{ICl}$. Of those who experienced a flare, 23 patients $(53 \%)$ were managed with topical therapy only; $16(21 \%)$ needed systemic therapy. Only five patients $(7 \%)$ required immunotherapy discontinuation for psoriasis flare. Forty-five patients (59\%) experienced other irAEs, $17(22 \%)$ of which were grade $3 / 4$. PFS with landmark analysis was significantly longer in patients with a psoriasis flare versus those without (39 vs 8.7 months, $p=0.049$ ).

Conclusions In this multicenter study, ICI therapy was associated with frequent psoriasis exacerbation, although flares were manageable with standard psoriasis treatments and few required ICI discontinuation. Patients who experienced disease exacerbation performed at least as well as those who did not. Thus, pre-existing psoriasis should not prevent patients from receiving ICls for treatment of malignancy.

\section{INTRODUCTION}

Immune checkpoint inhibitors (ICIs) block inhibitory T-cell receptors and promote increased anti-tumor activity. ${ }^{1}$ These agents, specifically antibodies targeting CTLA-4, PD-1, and PD-L1, have transformed cancer treatment and are now approved in over 15 different cancers. ${ }^{1-6}$ Approximately $43.6 \%$ and $12.5 \%$ of patients with advanced malignancies are estimated to be eligible for and respond to ICI treatment, respectively. ${ }^{7}$

However, this increased T-cell activation also contributes to immune-related adverse events (irAEs), which most commonly affect the gut, skin, liver, lungs, and endocrine glands. ${ }^{18}$ Given concern for irAEs, initial trials excluded patients with pre-existing autoimmune diseases, which affect $3 \%-5 \%$ of the US population, and likely a higher proportion of patients with cancer. $^{9-11}$ Subsequent retrospective analyses have demonstrated that ICIs in this population have similar effectiveness to clinical trial populations. Additionally, an acceptable safety profile in this population has been suggested, although exacerbation of baseline autoimmune disease and perhaps modestly increased rates of traditional irAEs may occur. $^{12-16}$

Psoriasis vulgaris is a common, immunemediated disease that impacts approximately $2 \%-3 \%$ of adults in the USA, although prevalence differs across geographic regions and ethnic groups. It most commonly affects the 
skin and joints, and the chronic, recurrent nature of the disease negatively impacts patient health and wellbeing. ${ }^{17}{ }^{18}$ Several patients with psoriasis were included in studies examining the safety of immunotherapy in patients with pre-existing autoimmune disease. However, since autoimmune disease constitutes a heterogeneous group of conditions encompassing over 80 diseases, the specific relevance to patients with psoriasis is unclear. ${ }^{9} 10$ Smaller studies and case reports have reported exacerbation of pre-existing psoriasis while on immunotherapy, but treatment outcomes of ICIs in this population remain largely unestablished. ${ }^{1919-21}$ 21-23

In this study, we assessed 76 patients with pre-existing psoriasis and various cancer types treated with ICIs from eight academic centers in the USA, Australia, and Germany to further establish the safety and effectiveness of ICIs in this population.

\section{METHODS \\ Patients}

De-identified medical data were collected from each participating institution's electronic health records. All patients with a prior diagnosis of psoriasis vulgaris and who had received at least one dose of an ICI (anti-CTLA-4, anti-PD-1, or anti-PD-L1 antibodies) for treatment of any cancer were included.

\section{Study design}

Baseline patient demographics were recorded, including gender, age, body mass index, cancer type and pathologic stage, mutations, brain metastases, prior treatments, absolute neutrophil count, absolute lymphocyte count, and serum lactate dehydrogenase level. Pre-existing psoriasis vulgaris was characterised by type, areas of involvement, duration of symptoms; presence of psoriatic arthritis, uveitis, or other extracutaneous disease manifestations; treatment for psoriasis prior to ICI; and diagnosis of other autoimmune diseases. Details of cancer therapy included ICI therapy, dose, and number of doses. Safety was assessed by outlining and describing psoriasis flares during treatment and their subsequent management, and presence of other irAEs (evaluated by the Common Terminology Criteria for Adverse Events, V.5.0) ${ }^{24}$ Efficacy was evaluated with treatment response (classified according to Response Evaluation Criteria in Solid Tumors 1.1), ${ }^{25}$ progression-free survival (PFS), and overall survival (OS). PFS and OS, for the entire population and advanced melanoma subpopulation, were calculated from date of ICI initiation to date of progression and date of death (or last available follow-up), respectively. A landmark analysis, measured from the time point of 1.5 months, was performed to evaluate PFS and OS in patients who experienced a psoriasis flare versus those who did not and in patients who experienced any irAE, including psoriasis exacerbation, versus those who did not.

\section{Statistical analysis}

Summaries of categorical and continuous variables were outlined with percentages and means, respectively. The
Kaplan-Meier method was used to estimate OS and PFS and the log-rank test was used to compare the differences between the flare and no flare groups. Wilcoxon rank-sum tests and $\mathrm{X}^{2}$ analyses were used to evaluate the continuous and categorical clinical variables in association with psoriasis flares, respectively. Multivariable logistic regression model was fitted to assess the independent effect of immunotherapy class on the risk of psoriasis flares and treatment discontinuation for toxicity, adjusting for age and gender, and prior psoriasis therapy. Missing covariate data were imputed with multiple imputation using $\mathrm{R}$ package 'mi'. Adjusted ORs are reported with 95\% CIs. IQR ORs for the continuous variables were computed to compare the third quartile with the first quartile for the variable.

\section{RESULTS}

\section{Patient demographics and pre-existing psoriasis}

Of 76 patients studied, $50(66 \%)$ were male with a median age of 67 years (range 25-92 years). Melanoma was the most frequent cancer represented $(\mathrm{N}=62,82 \%)$; others consisted of non-small cell lung cancer, ${ }^{5}$ head and neck cancers, ${ }^{2}$ esophageal adenocarcinoma, ${ }^{2}$ and others. ${ }^{5}$ Fifty-one patients $(67 \%)$ received anti-PD-1/anti-PD-L1, 8 $(11 \%)$ anti-CTLA-4, and 17 (22\%) combination of antiPD-1/CTLA-4 blockade (table 1). Twenty-one (28\%) patients had stage III disease, including $9(12 \%)$ treated with adjuvant or neoadjuvant intent, and $55(72 \%)$ with stage IV disease, including $1(1 \%)$ treated with adjuvant intent. Characteristics of patients with advanced melanoma (ie, not treated with adjuvant or neoadjuvant intent) are detailed separately (online supplemental table 1).

Types of pre-existing psoriasis included plaque psoriasis (46 patients $(61 \%))$ and less frequently psoriasis guttate, pustular or psoriatic arthritis only (table 1 ). Prior to ICI treatment, the median duration of psoriasis symptoms was 9 years, although the duration of symptoms was unspecified or unknown in most patients $(57 \%)$. Psoriatic arthritis, as defined by each contributing center, was present in 15 patients (20\%); other extracutaneous disease associations linked to psoriasis including uveitis/iritis, inflammatory bowel disease, and cardiovascular disease were present in $13 \%(\mathrm{~N}=10)$. Forty-one patients $(54 \%)$ had received prior treatment for psoriasis, including 24 patients $(36 \%)$ with topical therapy only. Only two patients $(3 \%)$ were on systemic immunosuppressants at ICI initiation.

\section{Safety}

After treatment with ICIs, 43 patients (57\%) experienced a flare of psoriasis at a median of 44 days after ICI initiation (range of 1-725 days). Cutaneous flare was observed in 39 patients $(51 \%)$. Exacerbation of extracutaneous manifestations including arthritis and iritis was reported in seven (9\%); three had both cutaneous and extracutaneous flares. All patients who experienced extracutaneous flares had extracutaneous disease (arthritis or iritis) prior 
Table 1 Baseline patient characteristics

\begin{tabular}{ll}
\hline Characteristic & $\begin{array}{l}\text { No of patients } \\
\text { (\%, N=76) }\end{array}$ \\
\hline Age, median (range) & $67(25-92)$ \\
\hline Male sex & $50(66)$ \\
\hline Cancer type & \\
\hline \multicolumn{1}{c}{ Melanoma } & $62(82)$ \\
\hline Lung & $5(7)$ \\
\hline Head and neck cancer & $2(3)$ \\
\hline Esophageal adenocarcinoma & $2(3)$ \\
\hline Other & $5(7)$
\end{tabular}

Cancer stage

III

$21(28)$

\begin{tabular}{|lc|}
\hline Adjuvant/neoadjuvant & $9(12)$ \\
\hline Non-adjuvant & $12(16)$ \\
\hline IV & $55(72)$ \\
\hline Adjuvant & $1(1)$ \\
\hline Non-adjuvant & $54(71)$ \\
\hline Immunotherapy class & $51(67)$ \\
\hline Anti-PD-1/PD-L1 & $8(11)$ \\
\hline Anti-CTLA-4 & $17(22)$ \\
\hline
\end{tabular}

Type of psoriasis

\begin{tabular}{|cc|}
\hline Plaque & $46(61)$ \\
\hline Guttate* & $4(5)$ \\
\hline Pustular & $2(3)$ \\
\hline Psoriatic arthritis only & $3(4)$ \\
\hline Not specified/unknown & $21(28)$ \\
\hline Psoriatic arthritis & $15(20)$ \\
\hline $\begin{array}{l}\text { Median duration of psoriasis symptoms, } \\
\text { years (range) }\end{array}$ & $9(1$ month-54 \\
\hline $\begin{array}{l}\text { Other extracutaneous disease } \\
\text { associations (including uveitis/iritis, IBD, }\end{array}$ & $10(13)$ \\
\hline CVD) & \\
\hline Prior psoriasis therapy & \\
\hline Acitretin & $1(1)$ \\
\hline Biologics $\dagger$ & $3(4)$ \\
\hline Methotrexateł & $6(8)$ \\
\hline Prednisone & $1(1)$ \\
\hline Small molecule inhibitors & $3(4)$ \\
\hline Topical therapy only & $27(36)$ \\
\hline None & $35(46)$ \\
\hline Active immunosuppressant psoriasis & $2(3)$ \\
therapy at start of immunotherapy & $6(8)$ \\
\hline Other pre-existing autoimmune disease & \\
\hline
\end{tabular}

Biologics include adalimumab and etanercept. Small molecule inhibitors include tofacitinib and apremilast.

*Includes two patients with guttate and plaque psoriasis.

†One patient on acitretin.

‡One patient on prednisone.

CVD, cardiovascular disease; IBD, inflammatory bowel disease. to ICI treatment. Of the 15 patients with baseline psoriatic arthritis, 6 experienced arthritis flares. Most psoriasis flares were grade 1 or 2 with only seven $(9 \%)$ noted as grade 3 or 4 . Among all patients, only five $(7 \%)$ discontinued ICI due to psoriasis flares. Multivariable analysis did not show an association of psoriasis flares with prior psoriasis therapy, age, gender, therapy class (anti-PD-1 vs anti-CTLA-4 vs combination blockade), or presence of psoriatic arthritis (online supplemental table 2).

Regarding treatment for psoriasis flares, of the 35 patients with cutaneous involvement only, 20 had improvement or resolution with topical therapies alone, including topical corticosteroids, calcipotriol, and phototherapy. Nine required additional treatments, including three with acitretin alone, two with prednisone alone (at unknown dose and $50 \mathrm{mg}$ ), one with acitretin and prednisone $10 \mathrm{mg}$, and one with apremilast and prednisone $7.5 \mathrm{mg}$, as well as two with antihistamines. Two patients with isolated cutaneous flare did not receive topical therapy and improved with apremilast only, and an additional patient improved with prednisone $50 \mathrm{mg}$ and acitretin. Three patients with cutaneous flare resolved without treatment.

Of the three patients with concurrent cutaneous and extracutaneous flares (two with grade 3 arthritis and one with grade 1 iritis), all were treated with topical agents. The two patients with grade 3 arthritis exacerbation also required additional systemic treatment, including one with prednisone $10 \mathrm{mg}$ and the other with prednisone $25 \mathrm{mg}$ and methotrexate. Four additional patients experienced an isolated flare of psoriatic arthritis, all grade 2, of which three improved with prednisone (at doses of 10, 25, and $25 \mathrm{mg}$ ) and one with non-steroidal anti-inflammatory drugs (NSAIDs).

Five patients $(7 \%)$, all with melanoma, required ICI discontinuation due to psoriasis flares. Of these, all patients received prior psoriasis therapy, and flares tended to occur early in ICI treatment (median of 14 days; range 1-703 days). Two had isolated cutaneous involvement (grades 3 and 4), two had arthritis only (grade 2), and one had both cutaneous and extracutaneous flares (grade 2 cutaneous, grade 3 arthritis). For their flares, one had topicals only, and four received prednisone, with one further needing apremilast and one requiring methotrexate.

Aside from psoriasis flares, other irAEs were observed in 45 patients $(59 \%)$, with grade $3-4$ irAEs in 17 (22\%) (table 2). In our population, grade 3-4 irAEs were observed at a rate of $16 \%$ for anti-PD-1/PD-L1, $37.5 \%$ for ipilimumab, and $35 \%$ for combination therapy. No patients had grade 5 (fatal) events. IrAEs were most commonly colitis (16 patients $(21 \%)$ ), skin toxicities excluding psoriasis $(13(17 \%))$, endocrinopathies $(11(14 \%))$, hepatitis (11 $(14 \%))$, and arthralgias not thought to represent psoriatic arthritis $(6(8 \%))$. Other irAEs resulted in ICI discontinuation in 22 patients (29\%). Multivariate logistic regression on factors associated with ICI discontinuation due to treatment-related toxicities revealed a significant 
Table 2 Psoriasis exacerbation and other IrAEs

\begin{tabular}{lc} 
Exacerbation information & $\begin{array}{l}\text { No of patients } \\
(\%, \mathbf{N}=76)\end{array}$ \\
\hline Experienced psoriasis flare & $43(57)$ \\
Cutaneous involvement & $39(51)$ \\
Extracutaneous manifestations & $7(9)$ \\
Arthritis & $6(8)$ \\
Iritis & $1(1)$
\end{tabular}

Time (days) from ICl initiation to flare, median (range)

\section{Worst grade of psoriasis flare}

$\begin{array}{cc}1 & 15(20) \\ 2 & 21(28) \\ 3 & 6(8) \\ 4 & 1(1)\end{array}$

Treatment for psoriasis flare

\begin{tabular}{|lc|}
\hline Acitretin* & $3(4)$ \\
\hline Methotrexate† & $1(1)$ \\
\hline Prednisone $\neq$ & $9(12)$ \\
\hline Small molecule inhibitors§ & $3(4)$ \\
\hline Topicals only & $23(30)$ \\
\hline Total patients with other irAE & $45(59)$ \\
\hline Colitis (including diarrhea) & $16(21)$ \\
\hline Skin (excluding psoriasis) & $13(17)$ \\
\hline Endocrine & $11(14)$ \\
\hline Liver & $11(14)$ \\
\hline Joint & $6(8)$ \\
\hline Lung & $3(4)$ \\
\hline Mucositis/oral cavity & $2(3)$ \\
\hline Other & $3(34)$ \\
\hline Grade 3 or 4 other irAE & $17(22)$ \\
\hline Colitis (including diarrhea) & $7(9)$ \\
\hline Skin (excluding psoriasis) & $2(3)$ \\
\hline Endocrine & 0 \\
\hline Liver & $5(7)$ \\
\hline Joint & $1(1)$ \\
\hline Lung & $2(3)$ \\
\hline Mucositis/oral cavity & 0 \\
\hline Other & 0 \\
\hline
\end{tabular}

Grade 3 or 4 other irAE per

immunotherapy class

$\begin{array}{ll}\text { Anti-PD-1/PD-L1 } & 8(16) \\ \text { Anti-CTLA-4 } & 3(37.5) \\ \text { Combination } & 6(35)\end{array}$

Reason for immunotherapy

discontinuation

Treatment completion/response/patient 17 (22) decision
Table 2 Continued

\begin{tabular}{|cc|}
\hline Exacerbation information & $\begin{array}{l}\text { No of patients } \\
(\%, \mathbf{N}=\mathbf{7 6})\end{array}$ \\
\hline Psoriasis flare & $5(7)$ \\
\hline Other irAE & $22(29)$ \\
Disease progression & $22(29)$ \\
Other (including ongoing treatment) & $10(13)$ \\
\hline
\end{tabular}

*Two patients treated with topicals.

†One patient treated with prednisone/topicals.

$\ddagger$ Three patients treated with topicals, one with acitretin, one with small molecule inhibitor/topicals.

§One patient treated with acitretin/topicals, one with topicals. $\mathrm{ICl}$, immune checkpoint inhibitor; irAEs, immune-related adverse events.

association between discontinuation due to ICI toxicity and combination anti-PD-1/CTLA-4 versus anti-PD-1 immunotherapy classes (online supplemental table 2). No association with ICI discontinuation was found for age, gender, or prior psoriasis therapy.

\section{Rechallenge}

Overall, 20 patients $(26 \%)$ were rechallenged with ICIs or received additional treatment with ICIs after their initial treatment regimen (online supplemental table 3). Among nine patients who received the same class of ICI, one flared. Among 11 patients who had different classes (eg, switched from combination PD-1/CTLA-4 blockade to anti-PD-1 monotherapy), 1 flared. Both psoriasis flares were grade $1-2$, and neither caused ICI discontinuation.

\section{Activity}

In patients with melanoma $(\mathrm{N}=62)$, median PFS was 39 months, and median OS was 87 months. Ten patients received ICIs as neoadjuvant or adjuvant treatment. Of 52 patients with advanced melanoma (ie, not treated with neoadjuvant or adjuvant intent), the response rate was $57.7 \%$ (20 complete response, 10 partial response), and additional four patients had stable disease (table 3). PFS and OS were compared between patients who experienced a psoriasis flare (cutaneous and/or extracutaneous) and those who did not. A landmark analysis at a time point of 1.5 months for PFS was completed since median time to flare was 44 days. In patients with advanced melanoma, median PFS was 43.8 months in the flare group vs 5.0 months in the no flare group $(\mathrm{p}=0.015)$ from a landmark analysis of 1.5 months; median OS for patients with melanoma was not reached in the flare group vs 29.3 months in the no flare group $(\mathrm{p}=0.024)$ (figure 1). PFS and OS were also compared between patients who experienced any irAE (including psoriasis flare) and those who did not for patients with advanced melanoma. Median PFS was 43.8 months in the irAE group vs 2.8 months in the no irAE group $(\mathrm{p}<0.001)$ from a landmark analysis of 1.5 months; median OS for patients 
Table 3 Psoriasis flares and $\mathrm{ICl}$ therapy response in patients with melanoma

\begin{tabular}{ll}
\hline Flares or ICI response details & $\begin{array}{l}\text { No with } \\
\text { melanoma } \\
(\%, \mathbf{N = 6 2 )}\end{array}$ \\
\hline $\begin{array}{l}\text { Experienced psoriasis flare (cutaneous and } \\
\text { extracutaneous) }\end{array}$ & $37(60)$ \\
\hline Grade 3 or 4 flare & $4(6)$ \\
Neoadjuvant or adjuvant treatment & $10(16)$ \\
\hline Non-adjuvant treatment & $52(84)$ \\
\hline Complete response & $20(32)$ \\
\hline Partial response & $10(16)$ \\
\hline Stable disease & $4(6)$ \\
\hline Progressive disease & $18(29)$ \\
\hline
\end{tabular}

$\mathrm{ICl}$, immune checkpoint inhibitor.

with melanoma was 87.3 months in the irAE group vs 17.1 months in the no irAE group $(\mathrm{p}=0.0006)$ (figure 2).

In an exploratory fashion, when examining all cancer types, 69 patients had evaluable responses, including 3 patients treated with neoadjuvant therapy. The response rate was $52.1 \%$ (23 CR, $13 \mathrm{PR}$ ) (online supplemental table 4); additional ninepatients had stable disease as best response. Median PFS was 20.0 months, and median OS was 87.3 months. PFS and OS were compared between patients who experienced a flare and those who did not for all cancer types. Median PFS was 39 months in the flare group vs 8.7 months in the no flare group $(p=0.049)$; median OS was not reached in the flare group vs 29.3 months in the no flare group $(p=0.045)$ (online supplemental figure 1). Longer time on therapy was significantly associated with presence of psoriasis flare $(\mathrm{p}=0.019) \quad$ (table 4). Notably, class of ICI and presence of prior psoriasis therapy were not associated with flares. PFS and OS were also compared between patients who had any irAE (including psoriasis flare) and those who did not. Median PFS was 39.0 months in the irAE group vs 3.4 months in the no irAE group $(\mathrm{p}=0.018)$; median OS was 87.3 in the irAE group vs 17.5 months in the no irAE group ( $p=0.028$ ) (online supplemental figure 2 ).

\section{DISCUSSION}

This multicenter investigation is the largest study to date exploring the use of ICIs in patients with pre-existing psoriasis. A Th1 response is thought to predominate in the pathogenesis of psoriasis, and cancer immunotherapy stimulates these helper T-cells, leading to concern about the safety of immunotherapy in patients with psoriasis. ${ }^{26} 27$ However, our study supports the safety of immunotherapy in this population. Although we observed a $57 \%$ rate of flares, most patients were successfully managed with traditional treatments, including topical therapies, systemic non-immunosuppressants, and rarely systemic immunosuppressants, and only $7 \%$ of patients required ICI discontinuation due to psoriasis flares. Over half of patients (59\%) experienced other irAEs, $22 \%$ of which were grade 3 or 4 . Overall, we observed excellent antitumor outcomes, and patients who experienced a psoriasis flare had improved PFS and OS compared with those who did not, although this could have been confounded by time on therapy.

Over half of patients $(57 \%)$ experienced exacerbation of psoriasis, seemingly higher than that previously reported with ICI treatment in autoimmune diseases in general. Previous reports have noted flares of baseline disease in $27 \%-47 \%$ of patients with autoimmune diseases treated with ICIs. ${ }^{12} 1428$ Small numbers of patients with psoriasis in these studies reported a wide range of rates

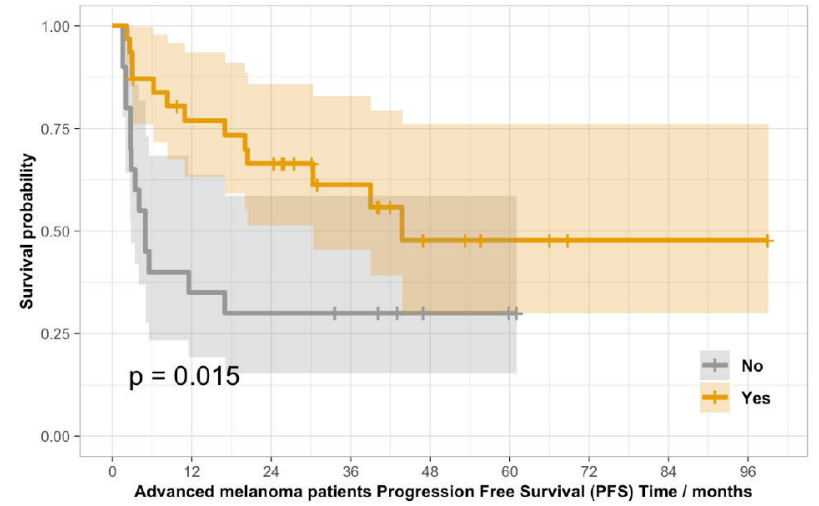

Number at risk

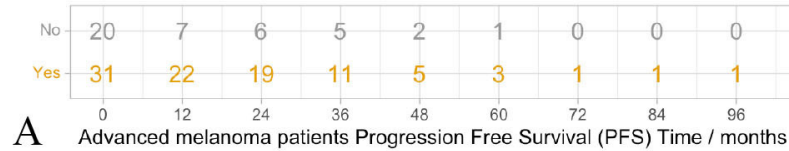

Figure 1 PFS (A) and OS (B), in months, of patients who experienced a psoriasis flare ('Yes') versus those who did not ('No') for all patients with melanoma with number of patients at risk and $95 \% \mathrm{Cls}$. Analysis based off a landmark analysis from time point 1.5 months. 


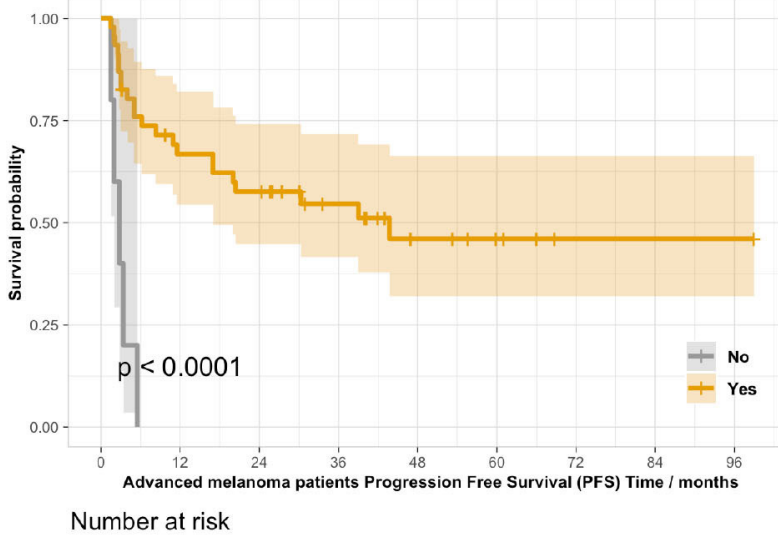

\begin{tabular}{c|ccccccccc|c|}
\hline & 5 & 0 & 0 & 0 & 0 & 0 & 0 & 0 & 0 \\
\hline 46 & 29 & 25 & 16 & 7 & 4 & 1 & 1 & 1 \\
\hline 0 & 12 & 24 & 36 & 48 & 60 & 72 & 84 & 96 \\
\hline
\end{tabular}

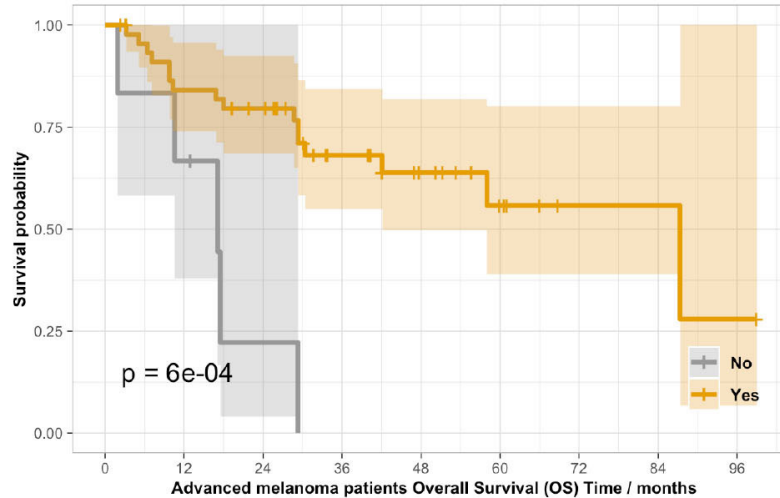

Number at risk

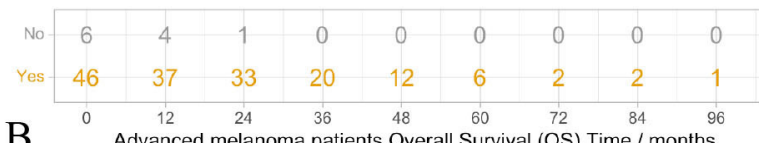

B Advanced melanoma patients Overall Survival (OS) Time/months

Figure 2 PFS (A) and OS (B), in months, of patients who experienced any irAE, including psoriasis flare, ('Yes') versus those who did not ('No') for all patients with melanoma with number of patients at risk and $95 \%$ Cls. irAE, immune-related adverse event.

of exacerbation (20\%-68\%) ${ }^{12-14} 28$ Our study provides a larger population across multiple centers and countries, thus potentially providing a more generalizable assessment of outcomes in this patient population.

Despite the high rate of flares, they were effectively managed by conventional treatments including topical therapies, non-immunosuppressives, and less often, systemic immunosuppressants. In fact, 27 patients $(63 \%$ of those who flared) were effectively managed with topical therapy alone or even did not need therapy. Escalation to systemic treatment, including acitretin, small molecule inhibitors, methotrexate, and prednisone, was required in 16 patients $(37 \%)$. Since $21 \%$ of flares needed prednisone treatment and only $7 \%$ of patients required ICI discontinuation due to flares, this study demonstrates frequent improvement in psoriasis exacerbation without use of treatment-impeding solutions, potentially offering guidance to clinicians facing these situations. This also suggests that dermatology consultation may facilitate ICI continuation, as suggested in other reports of ICIassociated skin toxicity. ${ }^{29}$ While cutaneous exacerbation was relatively manageable, arthritis flares more commonly required systemic immunosuppression, with five of six patients requiring prednisone, which may impact treatment decisions in patients with extracutaneous disease at baseline.

Compared with studies assessing patients with other pre-existing autoimmune disorders, we found a similar rate of other irAEs (59\%), including 22\% with grade 3 or 4 irAEs and no fatal events. This included grade 3-4 events in $16 \%$ of those receiving anti-PD-1/PD-L1, $37.5 \%$ for ipilimumab, and $35 \%$ for combination therapy, broadly similar to clinical trial populations. ${ }^{241214}$ Although these subgroups are relatively small, the aggregate data suggest an acceptable safety profile across classes of ICI.

While not a direct comparison, PFS and OS of our entire population (median 20.0 and 87.3 months, respectively) compare very favorably with previously reported survival data in phase 3 clinical trials for ICIs and in studies on patients with varying cancer types and autoimmune disease. ${ }^{1-41228}$ Additionally, PFS and OS of patients with melanoma (median 39 and 87 months, respectively) seem to exceed survival reported in initial phase 3 trials on antiPD-1 and anti-CTLA-4 antibodies in melanoma. ${ }^{23}$ Given the tolerable safety profile and apparent effectiveness of ICI in patients with psoriasis, ICIs remain a valuable

Table 4 Associations of clinical variables with psoriasis flares

\begin{tabular}{lccc}
\hline & Patients with psoriasis flares & Patients without psoriasis flares & P value \\
\hline Immunotherapy class & & & 0.27 \\
Anti-PD-1/PD-L1 & $32(42 \%)$ & $19(25 \%)$ & $5(7 \%)$ \\
Anti-CTLA-4 & $3(4 \%)$ & $9(12 \%)$ & 0.077 \\
Combination PD-1/CTLA-4 blockade & $8(11 \%)$ & & \\
Psoriasis therapy & & $14(42 \%)$ & 0.019 \\
$\quad$ Prior psoriasis therapy & $27(63 \%)$ & $19(58 \%)$ & 63 days \\
$\quad$ No prior psoriasis therapy & $16(37 \%)$ & & \\
Time on therapy & 168 days & & \\
\hline
\end{tabular}


treatment option for cancer in this population. Although monitoring for baseline disease exacerbation and other irAEs should continue while on ICI, patients with psoriasis should not be deprived access to ICI therapy. ${ }^{30}$

In terms of survival, patients who experienced psoriasis exacerbations appeared to perform at least as well as patients who did not experience disease flares, with significantly longer PFS and OS in patients with flares. Since some classic irAEs could conceivably be related to psoriasis (non-psoriaform skin eruptions, arthritis, and colitis), we also assessed PFS and OS for patients with all types of irAEs and found similar results. Conflicting evidence exists in the literature about the association between immunotoxicity and survival outcomes, with some studies reporting positive association between presence of immune events and survival and others without strong associations. ${ }^{31-33}$ However, we observed a statistically significant correlation between increasing time on therapy and flare likelihood. ${ }^{14}{ }^{34}$ This could create confounding, as longer time on therapy also correlates with treatment benefit (as progressing patients are taken off therapy) as well as psoriasis flares, although the landmark analysis from 1.5 months helps account for the potential impact of this lead time bias. Our results suggest patients who experienced a flare or irAE performed at least comparably to those who did not, including some patients with flares who needed to pause or permanently stop therapy or receive high doses of immunosuppression.

This study has several limitations. Although large compared with other studies on autoimmune disease and specifically psoriasis, the sample size of this study is still small. ${ }^{12-14} 28$ Larger studies on this population are needed to better analyze predictors of flare and clinical outcomes. Additionally, our data collection relied on details in the medical record, primarily from oncology documentation rather than systematic dermatology evaluations. Given the largely qualitative nature of describing psoriasis, documentation often differed across patients and institutions, and we lacked more detailed, objective measures of psoriasis severity, such as Psoriasis Area and Severity Index (PASI) scores, hindering the quantitative assessment of baseline psoriasis and disease flares. While a limitation, this lack of detailed information reflects what is often encountered by oncologists in real-world practice. Lastly, the specificity of psoriasis flare characterisation may be limited, as psoriasis flares in the skin and joints may be difficult to distinguish from classic ICI skin and joint toxicity, and we relied on each center's differentiation between a psoriasis flare and the classic lichenoid skin rash that may occur with immunotherapy.

In conclusion, our study is the largest assessing the impact of ICIs on patients with pre-existing psoriasis. Although flares were frequent, they tended to be low grade, were managed with standard psoriasis therapies, and rarely caused ICI discontinuation. This population had excellent survival outcomes, and the association of psoriasis flares with improved outcomes should be explored further. While it may require additional multidisciplinary management, these data indicate that psoriasis should not generally preclude treatment of advanced melanoma with immunotherapy.

\section{Author affiliations}

${ }^{1}$ Vanderbilt University School of Medicine, Nashville, Tennessee, USA

${ }^{2}$ Memorial Sloan Kettering Cancer Center, New York, New York, USA

${ }^{3}$ Alfred Hospital, Melbourne, Victoria, Australia

${ }^{4}$ Department of Medical Oncology, Alfred Hospital, Melbourne, Victoria, Australia ${ }^{5}$ Department of Medical Oncology, Peter MacCallum Cancer Centre, Melbourne, Victoria, Australia

${ }^{6}$ Department of Dermatology, Vanderbilt University Medical Center, Nashville,

Tennessee, USA

${ }^{7}$ Department of Biostatistics, Vanderbilt University Medical Center, Nashville, Tennessee, USA

${ }^{8}$ Department of Dermatology, NCT, University Hospital Heidelberg, Heidelberg, Germany

${ }^{9}$ Department of Medicine, Division of Hematology/Oncology, Northwestern University Feinberg School of Medicine, Chicago, Illinois, USA

${ }^{10}$ Rutgers Robert Wood Johnson Medical School, Piscataway, New Jersey, USA

${ }^{11}$ Massachusetts General Hospital Cancer Center, Boston, Massachusetts, USA

${ }^{12}$ Department of Dermatology, Massachusetts General Hospital, Boston,

Massachusetts, USA

${ }^{13}$ Melanoma Institute Australia, North Sydney, New South Wales, Australia

${ }^{14}$ Department of Dermatology, University Hospital Zurich, Zurich, Switzerland

${ }^{15}$ Faculty of Medicine and Health, The University of Sydney, Sydney, New South

Wales, Australia

${ }^{16}$ Westmead Hospital, Westmead, New South Wales, Australia

${ }^{17}$ Department of Medicine, Division of Hematology/Oncology, Vanderbilt University Medical Center, Nashville, Tennessee, USA

Contributors All authors have read and approved of this submission.

Funding National Cancer Institute (R01CA227481).

Competing interests DBJ has served on advisory boards for BMS, Catalyst Biopharma, lovance, Jansen, Merck, Novartis, Oncosec, and Pfizer; and received research funding from BMS and Incyte. JCH declares research support from Bristol Myers Squibb; advisory board honoraria from Pierre Fabre, Sanofi, Sun Pharma and Merck Sharp \& Dome; speakers honoraria from Bristol Myers Squibb, Merck Sharp \& Dohme, Novartis, Roche, Sanofi and Almirall; and travel support from Pierre Fabre. AM has served on advisory boards for BMS, MSD, Novartis, Roche, Pierre-Fabre and QBiotics. GL is consultant advisor for Aduro Biotech, Amgen, Array Biopharma, Boehringer Ingelheim International, Bristol-Myers Squibb, Highlight Therapeutics, Merck Sharpe \& Dohme, Novartis Pharma, Pierre Fabre, QBiotics Group Limited, and Regeneron Pharmaceuticals. PB declares travel support from MSD; and advisory board honoraria from Novartis. MC is a consultant advisor for Amgen, BMS, Eisai, Ideaya, MSD, Nektar, Novartis, Oncosec, Pierre-Fabre, Qbiotics, Regeneron, Roche, Provectus and Sanofi. AH has served on advisory boards for BMS, MSD, Novartis, Pierre-Fabre and QBiotics. MEL consults with Johnson and Johnson, Novocure, QED, Bicara, Janssen, Novartis, F Hoffmann-La Roche, EMD Serono, AstraZeneca, Innovaderm, Deciphera, DFB, Azitra, Kintara, RBC/La Roche Posay, Trifecta, Varsona, Genentech, Loxo, Seattle Genetics, Lutris, OnQuality, Azitra, Roche, Oncoderm, NCODA, and Apricity. MEL receives research funding from Lutris, Paxman, Novocure, J\&J, US Biotest, OQL, Novartis and AZ; and is funded in part through $\mathrm{NIH/NCI}$ Cancer Center Support Grant P30 CA008748. MJM has served as a consultant and/or received honorarium from AstraZeneca Pharmaceuticals, Catalyst Pharmaceuticals, Nektar Therapeutics and Immunai. STC serves on the Pfizer Advisory Board on digital media and the BOD for Medical Dermatology Society and is the Chair for Organisational Structure Committee of the AAD. ABW and VMR are supported by the NIH/NCl Cancer Center Support Grant P30 CA008748. VMR is funded by the Melanoma Research Alliance Young Investigator Award 614197 and an expert advisor for Inhabit Brands, Inc.

Patient consent for publication Not required.

Ethics approval Institutional review board approval was received for study protocols from each institution.

Provenance and peer review Not commissioned; externally peer reviewed

Data availability statement No data are available. All data relevant to the study are included in the article or uploaded as supplemental information. 
Supplemental material This content has been supplied by the author(s). It has not been vetted by BMJ Publishing Group Limited (BMJ) and may not have been peer-reviewed. Any opinions or recommendations discussed are solely those of the author(s) and are not endorsed by BMJ. BMJ disclaims all liability and responsibility arising from any reliance placed on the content. Where the content includes any translated material, BMJ does not warrant the accuracy and reliability of the translations (including but not limited to local regulations, clinical guidelines, terminology, drug names and drug dosages), and is not responsible for any error and/or omissions arising from translation and adaptation or otherwise.

Open access This is an open access article distributed in accordance with the Creative Commons Attribution Non Commercial (CC BY-NC 4.0) license, which permits others to distribute, remix, adapt, build upon this work non-commercially, and license their derivative works on different terms, provided the original work is properly cited, appropriate credit is given, any changes made indicated, and the use is non-commercial. See http://creativecommons.org/licenses/by-nc/4.0/.

\section{ORCID iDs}

Briana Rose Halle http://orcid.org/0000-0001-8311-617X

Allison Betof Warner http://orcid.org/0000-0001-6422-2997

Nicholas R Kurtansky http://orcid.org/0000-0002-6745-0386

Ryan Sullivan http://orcid.org/0000-0001-5344-6645

Meghan J Mooradian http://orcid.org/0000-0002-8289-8015

\section{REFERENCES}

1 Hodi FS, O'Day SJ, McDermott DF, et al. Improved survival with ipilimumab in patients with metastatic melanoma. $N$ Engl $J$ Med 2010;363:711-23.

2 Larkin J, Chiarion-Sileni V, Gonzalez R, et al. Combined nivolumab and ipilimumab or monotherapy in untreated melanoma. $N$ Engl $J$ Med 2015;373:23-34.

3 Larkin J, Chiarion-Sileni V, Gonzalez R, et al. Five-year survival with combined nivolumab and ipilimumab in advanced melanoma. $N$ Engl $J$ Med 2019;381:1535-46.

4 Robert C, Schachter J, Long GV, et al. Pembrolizumab versus ipilimumab in advanced melanoma. N Engl J Med 2015;372:2521-32.

5 Thallinger C, Füreder T, Preusser M, et al. Review of cancer treatment with immune checkpoint inhibitors. Wien Klin Wochenschr 2018;130:85-91.

6 Vaddepally RK, Kharel P, Pandey R, et al. Review of indications of FDA-approved immune checkpoint inhibitors per NCCN guidelines with the level of evidence. Cancers 2020;12 doi:10.3390/ cancers 12030738

7 Haslam A, Prasad V. Estimation of the percentage of US patients with cancer who are eligible for and respond to checkpoint inhibitor immunotherapy drugs. JAMA Netw Open 2019;2:e192535.

8 Johnson DB, Chandra S, Sosman JA. Immune checkpoint inhibitor toxicity in 2018. JAMA 2018;320:1702-3.

9 Hayter SM, Cook MC. Updated assessment of the prevalence, spectrum and case definition of autoimmune disease. Autoimmun Rev 2012;11:754-65.

10 Cooper GS, Stroehla BC. The epidemiology of autoimmune diseases. Autoimmun Rev 2003;2:119-25.

11 Khan SA, Pruitt SL, Xuan L, et al. Prevalence of autoimmune disease among patients with lung cancer: implications for immunotherapy treatment options. JAMA Oncol 2016;2:1507-8.

12 Johnson DB, Sullivan RJ, Ott PA, et al. Ipilimumab therapy in patients with advanced melanoma and preexisting autoimmune disorders. JAMA Oncol 2016;2:234-40.

13 Danlos F-X, Voisin A-L, Dyevre V, et al. Safety and efficacy of anti-programmed death 1 antibodies in patients with cancer and pre-existing autoimmune or inflammatory disease. Eur $\mathrm{J}$ Cancer 2018;91:21-9.
14 Tison A, Quéré G, Misery L, et al. Safety and efficacy of immune checkpoint inhibitors in patients with cancer and preexisting autoimmune disease: a nationwide, multicenter cohort study. Arthritis Rheumatol 2019;71:2100-11.

15 Brown LJ, Weppler A, Bhave P, et al. Combination anti-PD-1 and ipilimumab (IPI) therapy in patients with advanced melanoma and pre-existing autoimmune disorders (AD). JCO 2020;38:10026.

16 Abu-Sbeih H, Faleck DM, Ricciuti B, et al. Immune checkpoint inhibitor therapy in patients with preexisting inflammatory bowel disease. J Clin Oncol 2020;38:576-83.

17 Rachakonda TD, Schupp CW, Armstrong AW. Psoriasis prevalence among adults in the United States. J Am Acad Dermatol 2014;70:512-6.

18 Langley RGB, Krueger GG, Griffiths CEM. Psoriasis: epidemiology, clinical features, and quality of life. Ann Rheum Dis 2005;64:ii18-23.

19 De Bock M, Hulstaert E, Kruse V, et al. Psoriasis vulgaris exacerbation during treatment with a PD-1 checkpoint inhibitor: case report and literature review. Case Rep Dermatol 2018;10:190-7.

20 Phadke SD, Ghabour R, Swick BL, et al. Pembrolizumab therapy triggering an exacerbation of preexisting autoimmune disease: a report of 2 patient cases. J Investig Med High Impact Case Rep 2016;4:2324709616674316.

21 Politi A, Angelos D, Mauri D, et al. A case report of psoriasis flare following immunotherapy: report of an important entity and literature review. SAGE Open Med Case Rep 2020;8:2050313X1989770.

22 Liebman T, Adams L, Alapati U. Palmoplantar exacerbation of psoriasis after nivolumab for lung cancer. $J$ Community Support Oncol 2017;15:106-8.

23 Bonigen J, Raynaud-Donzel C, Hureaux J, et al. Anti-PD1-induced psoriasis: a study of 21 patients. J Eur Acad Dermatol Venereol 2017;31:e254-7.

24 Common terminology criteria for adverse events (CTCAE) version 5, 2017US Department of Health and Human Services, National Institutes of Health, National Cancer Institute. Available: https://ctep. cancer.gov/protocoldevelopment/electronic_applications/ctc.htm\# ctc 50 .

25 Eisenhauer EA, Therasse P, Bogaerts J, et al. New response evaluation criteria in solid tumours: revised RECIST guideline (version 1.1). Eur J Cancer 2009;45:228-47.

26 Knutson KL, Disis ML. Tumor antigen-specific T helper cells in cancer immunity and immunotherapy. Cancer Immunol Immunother 2005;54:721-8.

27 Griffiths CE, Barker JN. Pathogenesis and clinical features of psoriasis. Lancet 2007;370:263-71.

28 Menzies AM, Johnson DB, Ramanujam S, et al. Anti-PD-1 therapy in patients with advanced melanoma and preexisting autoimmune disorders or major toxicity with ipilimumab. Ann Oncol 2017;28:368-76.

29 Chen ST, Molina GE, Lo JA, et al. Dermatology consultation reduces interruption of oncologic management among hospitalized patients with immune-related adverse events: a retrospective cohort study. J Am Acad Dermatol 2020;82:994-6.

30 Johnson DB, Reynolds KL, Sullivan RJ, et al. Immune checkpoint inhibitor toxicities: systems-based approaches to improve patient care and research. Lancet Oncol 2020;21:e398-404.

31 Haratani K, Hayashi H, Chiba $\mathrm{Y}$, et al. Association of immune-related adverse events with nivolumab efficacy in non-small-cell lung cancer. JAMA Oncol 2018;4

32 Quach HT, Dewan AK, Davis EJ, et al. Association of antiprogrammed cell death 1 cutaneous toxic effects with outcomes in patients with advanced melanoma. JAMA Oncol 2019;5:906-8.

33 Das S, Johnson DB. Immune-related adverse events and anti-tumor efficacy of immune checkpoint inhibitors. J Immunother Cancer 2019;7:306.

34 Kähler KC, Eigentler TK, Gesierich A, et al. Ipilimumab in metastatic melanoma patients with pre-existing autoimmune disorders. Cancer Immunol Immunother 2018;67:825-34. 